\title{
PROGRESS IN THREE-DIMENSIONAL NON-DESTRUCTIVE ASSESSMENT OF REAL CRACKS FROM EDDY CURRENT TESTING SIGNALS
}

Enhancement in three-dimensional non-destructive evaluation of real cracks from two-dimensional simulated eddy current testing signals is reported in the paper. A new uniform eddy current testing probe is employed for the inspection. All three spatial components of the perturbation electromagnetic field are acquired during two-dimensional scan of the probe. The ECT signals due to partially conductive cracks are simulated by a fast forward FEM-BEM solver using a database. Two crack models are proposed for the inversion using the tabu search stochastic method. The former model has a cuboid shape and the latter one reflects a more complex geometry. Numerical results of the three-dimensional reconstruction of partially conductive cracks from simulated two-dimensional signals are presented and discussed in the paper.

Keywords: Non-destructive evaluation, eddy currents, partially conductive cracks, diagnosis, stochastic method, tabu search.

\section{Introduction}

New approaches such as System Health Monitoring and Condition Based Maintenance are nowadays employed for assessment of structural integrity of various components and structures. The modern methods follow three consecutive phases - detection of non-homogeneities, their diagnosis and prognosis of their further development. The first two phases are inherently associated with Non-Destructive Evaluation (NDE) of materials. Enhancing NDE methods is therefore very important for accomplishing their challenging missions.

NDE techniques are based on numerous physical principles and phenomena. Eddy current testing (ECT) is one of the widely utilized electromagnetic NDE methods. ECT works based on an interaction of time-varying electromagnetic field with a conductive body according to the Faraday's electromagnetic induction law. There are many advantages such as high sensitivity for surface breaking defects, high inspection speed, contact-less inspection, versatility, maturity of numerical means that account for continuously enlarging application area of the ECT mainly in nuclear, petrochemical and aviation industries [1]. On the other hand, ECT is a relative method and the inverse problem is ill-posed [2]. Therefore, evaluating dimensions of a detected defect from ECT response signals can be quite difficult [3]. ECT instruments provide raw data with limited or absent capability of interpreting quantitatively the data [4]. Typically, evaluation relies on calibrated curves measured on pre-fabricated etalons and on the skills of an operator. Recently, the progress in powerful computers has allowed developing of automated procedures to make decisions. Quite satisfactory results are reported by several groups for automated evaluation of artificial slits [3] and even for several parallel notches [5]. However, evaluation of real cracks, especially stress corrosion cracking (SCC), from ECT response signals remains still very difficult.

SCCs are quite different comparing to artificial slits or even to other types of real defects. Cross sections of SCC frequently show branched structure and group of cracks usually occur in what is known as a colony. The local opening of SCC is usually very small, e.g. tens of micrometers; however a damaged region itself is much broader. SCC contains many unbroken ligaments both in depth and opening directions, which makes SCC partially conductive [6]. In the case of artificial electro-discharge machined (EDM) notches, the width is usually considered fixed in the inversion process of ECT signals. However, for cracks with nonzero conductivity the width affects the signal and it has to be considered unknown during reconstruction [7]. It means that the additional variable should be taken into account for evaluation of a detected SCC which considerably increases ill-posedness of the inverse problem [2]. Thus, many unsatisfactory results are reported when the automated procedures originally developed for non-conductive cracks are employed in the evaluation of SCCs. It is stated that one of the possible reasons is lack of sufficient information [3].

\footnotetext{
* ${ }^{1}$ Ladislav Janousek, ${ }^{2}$ Mihai Rebican, ${ }^{1}$ Milan Smetana, ${ }^{2}$ Anton Duca

${ }^{1}$ Department of Electromagnetic and Biomedical Engineering, Faculty of Electrical Engineering, University of Zilina, Slovakia

${ }^{2}$ Faculty of Electrical Engineering, University Politehnica of Bucharest, Bucharest, Romania

E-mail: ladislav.janousek@fel.uniza.sk
} 
Common eddy current probes are designed in such a way that they sense only one component of the perturbation electromagnetic field. Usually, the most significant component is used for the evaluation. However, curved paths of eddy currents provide more information in principle.

Several studies of authors focused on enhancing information level of eddy current testing signals [8] - [10]. The original idea is based on sensing of all three spatial components of the perturbation electromagnetic field. The studies have revealed that the uncertainty in cracks' evaluation is reduced when the enriched information is utilized for the purpose. Promising results create new challenges concerning development of automatic procedures for diagnosis of real cracks.

The authors have already developed an algorithm for reconstruction of multiple slits from ECT signals by means of a stochastic optimization method, such as tabu search [5]. The reconstruction of multiple slits was done in three-dimensions. Therefore, the scheme is also appropriate for reconstruction of a partially conductive crack when the width has to be considered as variable.

This paper presents enhanced procedure for diagnosis of real cracks from simulated eddy current testing response signals. The tabu search is applied for the three-dimensional reconstruction of partially conductive cracks. Uniqueness of the proposal lies in the utilization of two-dimensional response signals for the $3 \mathrm{D}$ inversion. Moreover, all the three spatial components of the perturbation electromagnetic field are taken as the response signals in order to tackle severe problem connected with diagnosis of partially conductive cracks.

\section{Numerical model}

A plate specimen having the electromagnetic parameters of a stainless steel SUS316L is inspected in this study. The specimen has a thickness of $t=10 \mathrm{~mm}$, a conductivity of $\sigma=1.35 \mathrm{MS} / \mathrm{m}$ and a relative permeability of $\mu_{r}=1$. A single surface breaking crack appears in the plate. It is modelled as a cuboid having different electromagnetic properties from the base material. Configuration of the plate (region $\Omega_{0}$ ) with the crack (region $\left.\Omega_{1}\right)$ is shown in Fig. 1. The crack region $\Omega_{1}\left(22 \times 2 \times 10 \mathrm{~mm}^{3}\right)$ is uniformly divided into a grid composed of $n_{x} \times n_{y} \times n_{z}(11 \times 5 \times 10)$ cells defining a possible crack geometry. The dimensions of each cell are $2.0 \times 0.4 \times 1.0 \mathrm{~mm}^{3}$.

A new eddy-current probe proposed by the authors is employed for the near-side inspection of the plate [10]. It consists of two circular exciting coils positioned apart from each other and oriented normally regarding the plate's surface. The circular coils are connected in series but magnetically opposite to induce uniformly distributed eddy currents in the plate. The exciting coils are supplied from a harmonic source with a frequency of $5 \mathrm{kHz}$ and the current density $1 \mathrm{~A} / \mathrm{mm}^{2}$. A detection system of the probe is composed of three small circular coils oriented along three axes perpendicularly to each other [8]. The detection system is located in the centre between the exciting coils to gain high sensitivity as the direct coupling between the exciting coils and the detectors is minimal at this position. Configuration of the new probe is shown in Fig. 2. Dimensions of the detecting coils are as follows: an inner diameter of $1.2 \mathrm{~mm}$, an outer diameter of $3.2 \mathrm{~mm}$ and a winding height of $0.8 \mathrm{~mm}$.

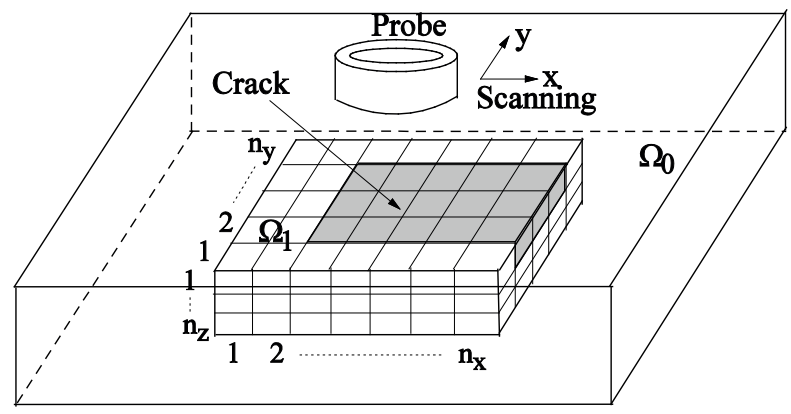

Fig. 1 Configuration of plate specimen with crack region

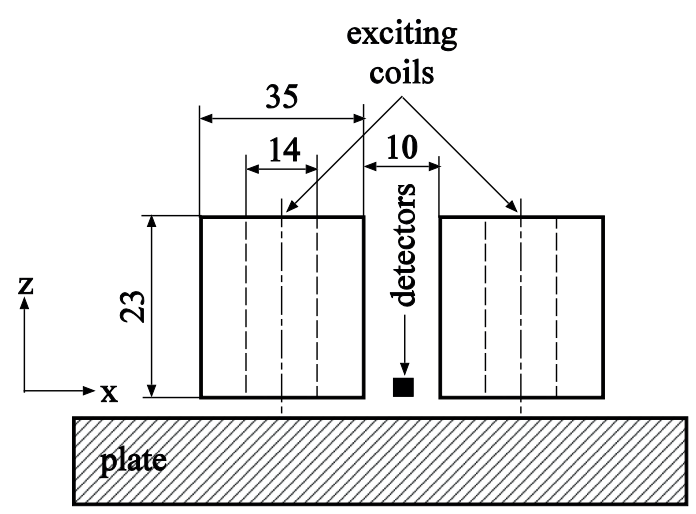

Fig. 2 ECT probe configuration

Two-dimensional scanning, so called C-scan, is performed over the cracked surface with a lift-off of $1 \mathrm{~mm}$. The real and imaginary parts of the induced voltages in all three detecting coils corresponding to three spatial components of the perturbation electromagnetic field are sensed and recorded during the inspection.

\section{Crack reconstruction}

The fast-forward FEM-BEM analysis solver using database [11] is adopted here for the ECT response signals simulation. Actually, a version of the database algorithm upgraded by the authors in previous works [5] for the computation of the ECT signals due to multiple cracks is used in this paper. The database is designed for a three-dimensional defect region and not as usually 
for a two-dimensional one where a crack width is considered fixed. Thus, the ECT response signals can be simulated also for partially conductive cracks with variable width using the same database generated in advance. The area of the simulated twodimensional ECT signals has surface dimensions of $100 \times 28 \mathrm{~mm}^{2}$. The number of scanning points in the two directions is 50 and 70 , respectively.

Tabu search is employed for the three-dimensional diagnosis of a detected crack [5]. The error function $\varepsilon$ to be minimized is defined as:

$$
\begin{aligned}
& \mathcal{E}=\frac{\sum_{i=1}^{n}\left|\Delta V_{i x}(c)-\Delta V_{i x}^{m}\right|^{2}}{\sum_{i=1}^{n}\left|\Delta V_{i x}^{m}\right|^{2}}+\frac{\sum_{i=1}^{n}\left|\Delta V_{i y}(c)-\Delta V_{i y}^{m}\right|^{2}}{\sum_{i=1}^{n}\left|\Delta V_{i y}^{m}\right|^{2}}+ \\
& +\frac{\sum_{i=1}^{n}\left|\Delta V_{i z}(c)-\Delta V_{i z}^{m}\right|^{2}}{\sum_{i=1}^{n}\left|\Delta V_{i z}^{m}\right|^{2}}
\end{aligned}
$$

where $c$ is the crack parameter vector, $\Delta V_{i}$ and $\Delta V_{i}^{m}$ are the simulated (reconstructed) and true (measured) induced pick-up voltages of the coils at the $i$-th scanning point respectively, and $n$ is the number of scanning points. It should be noted that the real and the imaginary parts of each spatial component (X, Y and $\mathrm{Z}$ according to the coordinate system shown in Figs. 1 and 2) of the perturbation electromagnetic field are employed for the inversion.

Two three-dimensional crack models shown in Fig. 3 are employed in the inversion. The partial conductivity of both crack models is considered uniform.

In the first model shown in Fig. 3a, the crack has a cuboid shape. The crack parameter vector $c$ consists of 6 integers, $c=\left[i x_{1}, i x_{2}, i y_{1}, i y_{2}, i z, s\right]$, where $i x_{1}$ and $i x_{2}$ are the indices of the first and last cells of the crack along the length of crack, $i y_{1}$ and $i y_{2}$ are the indices of the first and last cells of the crack along the width of crack, $i z$ is the number of cells of the crack along the depth of crack, and $\sigma_{c}=s \% \sigma\left(\sigma_{c}\right.$ - the conductivity of crack, $\sigma$ - the conductivity of base material). In Fig. 3a, for a uniform grid with $13 \times 5 \times 10$ cells, the parameter vector is $c=[6,13,1,3,4$ and 20 ]. Thus, $8 \times 3 \times 4$ cells form the crack, and the crack conductivity is $\sigma_{c}=20 \% \sigma$.

The second crack model shown in Fig. $3 \mathrm{~b}$ adopts a more complex shape. The crack depth is considered as variable along the crack length. The crack parameter vector $c$ consists of $n_{x}+3$ integers, $c=\left[i z_{1}, i z_{2}, \ldots, i z_{n x}, i y_{1}, i y_{2}, s\right]$, where $i z_{k}, k=1, n x$ is the number of cells of the crack along the depth of crack, $i y_{1}$ and $i y_{2}$ are the indices of the first and last cells of the crack along the width of crack, and $\sigma_{c}=s \% \sigma\left(\sigma_{c}\right.$ - the conductivity of crack, $\sigma$ - the conductivity of base material). In Fig. 3b, for a uniform grid with $13 \times 5 \times 10$ cells, the parameter vector contains 16 integers, as $c=[0,0,0,0,0,8,4,1,2,5,3,6,4,1,3,30]$. Thus, $(8+4+1+2+5+3+6+4) \times 3$ cells form the crack, and the crack conductivity is $\sigma_{c}=30 \% \sigma$.
In the both models, the cracks have the same orientation. The width of crack can have the values: 0.4, 0.8, 1.2, 1.6, $2 \mathrm{~mm}$.

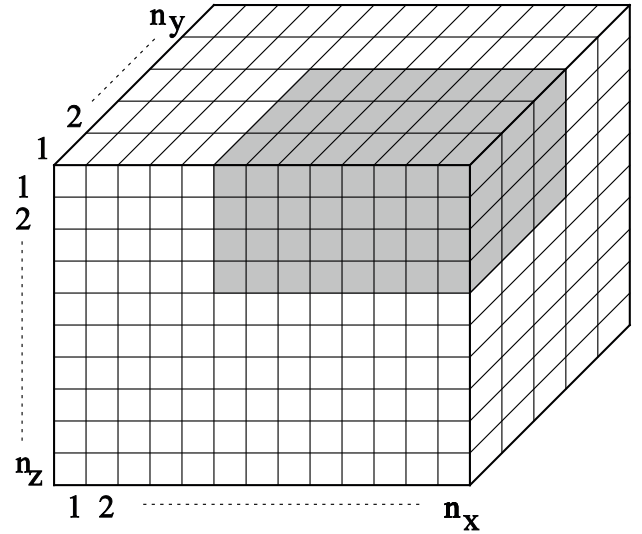

a) cuboid shape of crack (model 1)

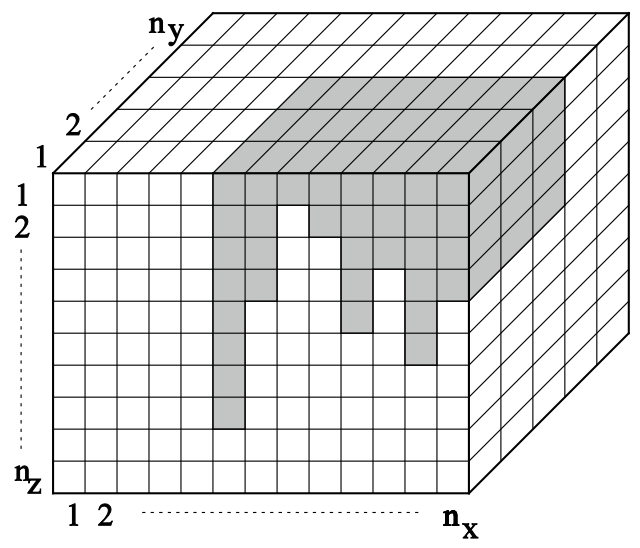

b) complex shape of crack (model 2)

Fig. 3 Crack region division

In this paper, the simulated signals are affected by added noise before the inversion process in order to prove the validity and robustness of the proposed approach. The perturbed signal is computed as:

$\left(\Delta V_{i}^{m}\right)_{m s}=\Delta V_{i}^{m}(1 \pm n s \%)$,

where $\Delta V_{i}^{m}$ and $\left(\Delta V_{i}^{m}\right)_{n s}$ are the initial and the perturbed true signals at the $i$-th scanning point respectively, $n s$ is a random value of an imposed maximum noise level.

Figure 4 shows the simulated ECT signal (Fig. 4a) and the perturbed ECT signal (Fig. 4b) for Z-component when a noise of maximum level $40 \%$ is added, for a partially conductive crack with a cuboid shape $\left(l_{c}=6 \mathrm{~mm}, w_{c}=0.8 \mathrm{~mm}, d_{c}=4 \mathrm{~mm}, s_{c}=5 \%\right.$ of $\left.\sigma\right)$. 


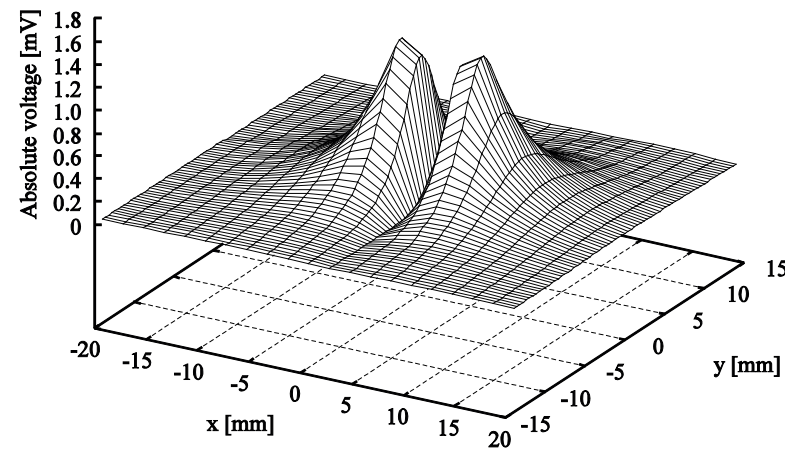

a) unperturbed simulated signal

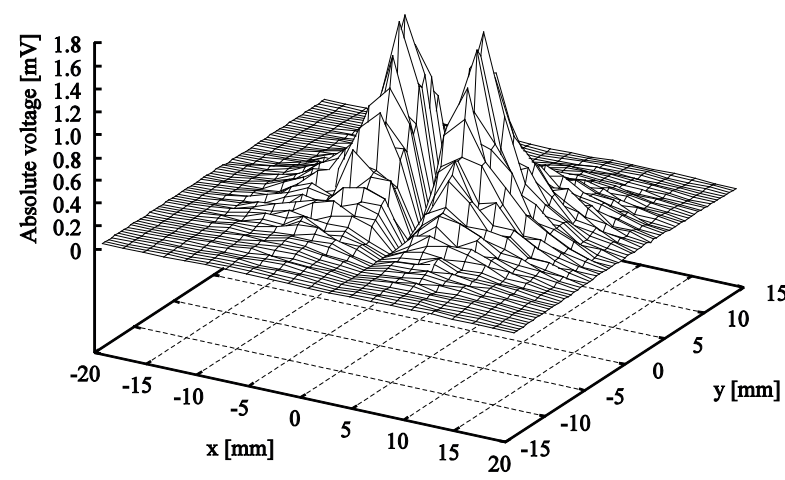

b) simulated signal perturbed with noise

Fig. 4 Simulated ECT response signal

\section{Results and discussions}

In Table 1 the numerical results of the reconstruction are presented when a partially conductive crack is modelled as a cuboid shape, Fig. 3a. The column denoted as "Real" gives the true dimensions $\left(l_{c} \times w_{c} \times d_{c}\right)$ and partial conductivity $\left(\sigma_{c}\right.$ in \% of $\sigma$ ) of the crack. The results of the diagnosis are provided in the column labelled as "Reconstructed" for various maximum levels of the noise added to simulated ECT signals. The error function $\varepsilon(c)$ of the diagnosis are reported, too. Five numerical simulations were performed for each crack reconstruction, with different random initializations of the tabu search initial population.

The time required for reconstruction of one crack is approximately $90-120$ minutes. When there is no noise added to signal, the reconstructed crack is the same with the real crack (the error function $\varepsilon=0$ ) for each random initial population. In the case of diagnosis from signals perturbed by noise (maximum noise level $=10,20,30,40 \%$ ), for the most random initial populations, the crack is exactly reconstructed even if the maximum level of noise is high (30,40\%). For one random initial population and for a perturbed signal by added noise of highest level, i.e. $40 \%$, the result is slightly different (the last column in
Reconstruction of partially conductive cracks, crack model 1 Table 1

\begin{tabular}{|l|r|r|r|r|r|r|r|}
\hline Crack & Real & \multicolumn{6}{|c|}{ Reconstructed } \\
\hline Noise (\%) & & \multicolumn{1}{|c|}{$\mathbf{0}$} & $\mathbf{1 0}$ & $\mathbf{2 0}$ & $\mathbf{3 0}$ & $\mathbf{4 0}$ & $\mathbf{4 0}$ \\
\hline $\boldsymbol{l}_{c}[\mathbf{m m}]$ & 6.0 & 6.0 & 6.0 & 6.0 & 6.0 & 6.0 & 6.0 \\
\hline $\boldsymbol{w}_{\boldsymbol{c}}[\mathbf{m m}]$ & 0.8 & 0.8 & 0.8 & 0.8 & 0.8 & 0.8 & 1.2 \\
\hline $\boldsymbol{d}_{c}[\mathbf{m m}]$ & 4.0 & 4.0 & 4.0 & 4.0 & 4.0 & 4.0 & 4.0 \\
\hline$\sigma_{c}[\mathbf{\%}]$ & 5 & 5 & 5 & 5 & 4 & 4 & 9 \\
\hline $\boldsymbol{\varepsilon} \cdot \mathbf{1 0}^{-\mathbf{3}}$ & - & 0 & 11 & 21 & 32 & 42 & 45 \\
\hline
\end{tabular}

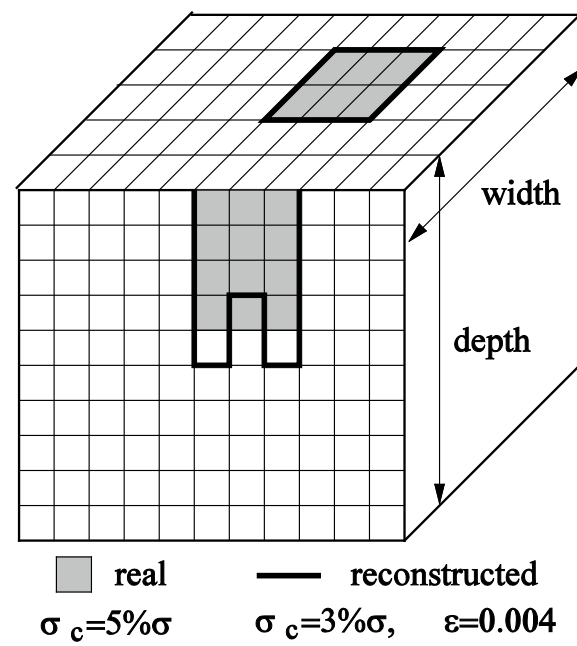

a) crack signal without noise

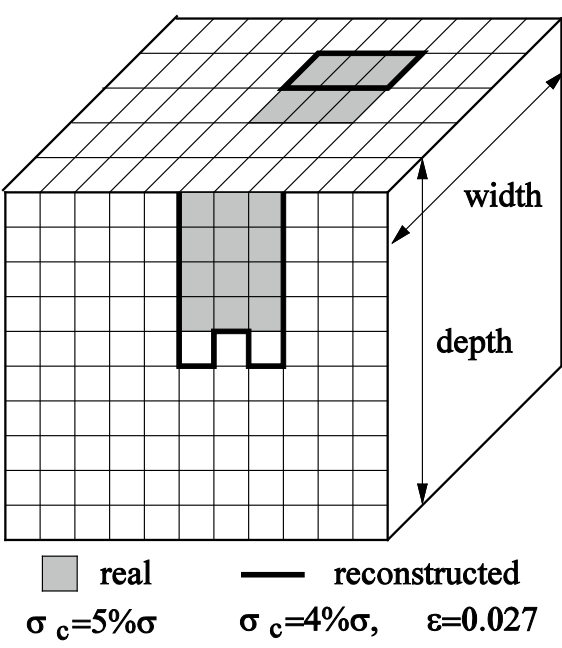

b) crack signal with added noise of $20 \%$

Fig. 5 Reconstruction of a partially conductive crack, crack model 2

Table 1): the length and depth are equal to the true values; the width and partial conductivity are estimated not exactly but with good precision (the width is different with the minimum value of $0.4 \mathrm{~mm}$ ). However, the last two parameters are not very important from structural integrity point of view. The results clearly show 
that the crack parameters are estimated quite precisely from the noisy ECT signals using the proposed approach for various random initial populations.

Figure 5 shows the results of three-dimensional diagnosis of the partially conductive crack described in Table 1 (column "Real") from 2D ECT signals without noise (Fig. 5a) and with added noise of maximum level $20 \%$ (Fig. 5 b) when the complex crack model (Fig. 3b) is employed for the inversion. One diagnosis takes around 5-7 hours. In the case of the reconstruction from signal without noise, the crack is precisely localized and also its length and width are exactly estimated. The depth profile does not perfectly copy the true one. However, the maximum depth is accurately assessed. But, for the reconstruction from signal with added noise of maximum level of $20 \%$, the width is smaller with a minimum value of $0.4 \mathrm{~mm}$ than the real width.

The presented results proved effectiveness of the enhanced approach of three-dimensional diagnosis of partially conductive cracks, even if cracks with complex shape and signals with added noise are considered. ECT response signals gained during C-scan together with acquiring all three spatial components of the perturbation electromagnetic field significantly improve the preciseness of inversion process using tabu search stochastic method.

\section{Conclusion}

Enhanced technique for three-dimensional diagnosis of partially conductive cracks was presented in the paper. A special eddy current probe driving uniformly distributed eddy currents was used for the inspection of a plate specimen. A detection system of the probe was designed in such a way that all three spatial components of the perturbation electromagnetic field were acquired. The tabu search stochastic method was employed for the reconstruction of partially conductive cracks profile from eddy current response signals gained during the $\mathrm{C}$-scan of the probe. Two crack models were proposed: a crack with cuboid shape and the other one with more complex shape. The length, depth, width and conductivity of the crack were considered unknown in the inversion process. The conductivity of the crack was uniform. The presented results proved that the proposed approach allows quite precisely reconstructing three-dimensional profile of a crack together with its partial conductivity from ECT response signals even with added noise.

Further work of the authors will concern more realistic shapes of cracks and validation with measured data from natural cracks (SCC).

\section{Acknowledgement}

This work was supported by the Slovak Research and Development Agency under the contracts No. APVV-0349-10 and APVV-0194-07.

This work was co-funded by a Slovakia-Romania joint grant by the Slovak Research and Development Agency under the contract No. SK-RO-0011-12 and the ANCS under the Grant No. 654/2013.

\section{References}

[1] JANOUSEK, L., MAREK, T., GOMBARSKA, D.: Eddy Current Non-destructive Evaluation of Conductive Materials, Communications - Scientific Letters of the University of Zilina, No. 1, pp. 29-33, 2006.

[2] YUSA, N., HUANG, H., MIYA, K.: Numerical Evaluation of the Ill-posedness of Eddy Current Problems to Size Real Cracks, NDT\&E International, vol. 40, pp. 185-191, 2007.

[2] JANOUSEK, L., SMETANA, M.: Uncertainty in Depth Evaluation of Partially Conductive Cracks from Eddy Current Testing Signals, Communications - Scientific Letters of the University of Zilina, vol. 13, pp. 55-60, 2011.

[4] RUBINACCI, G., TAMBURINO, A., VENTRE, S.: Fast Numerical Techniques for Electromagnetic Nondestructive Evaluation, Nondestructive Testing and Evaluation, vol. 24, pp. 165-194, 2009.

[5] REBICAN, M., CHEN, Z., YUSA, N., JANOUSEK, L., MIYA, K.: Shape Reconstruction of Multiple Cracks from ECT Signals by Means of a Stochastic Method, IEEE Transactions on Magnetics, vol. 42, pp. 1079-1082, 2006.

[6] YUSA, N., MIYA, K.: Discussion on the Equivalent Conductivity and Resistance of Stress Corrosion Cracks in Eddy Current Simulations, NDT\&E International, vol. 42, pp. 9-15, 2009.

[7] CHEN, Z., REBICAN, M., YUSA, N., MIYA, K.: Fast Simulation of ECT Signal Due to a Conductive Crack of Arbitrary width, IEEE Transactions on Magnetics, vol. 42, pp. 683-686, 2006.

[8] JANOUSEK, L., SMETANA, M., CAPOVA, K.: Enhancing Information Level in Eddy-current Non-destructive Inspection, Intern. J. of Applied Electromagnetics and Mechanics, vol. 33, pp. 1149-1155, 2010.

[9] JANOUSEK, L., SMETANA, M., ALMAN, M.: Decreasing Uncertainty in Size Estimation of Stress Corrosion Cracking from Eddy-current Signals, Studies in Applied Electromagnetics and Mechanics, vol. 35, pp. 53-60, 2011. 


\section{coMMNICOIIONS}

[10] JANOUSEK, L., SMETANA, M., ALMAN, M.: Decline in Ambiguity of Partially Conductive Cracks' Depth Evaluation from Eddy Current Testing Signals, Intern. J. of Applied Electromagnetics and Mechanics, vol. 39, pp. 329-334, 2012.

[11] CHEN, Z., MIYA, K., KUROKAWA: Rapid Prediction of Eddy Current Testing Signals Using A - $\phi$ Method and Database, NDT\&E International, vol. 32, pp. 29-36, 1999. 